\title{
In-Silico Molecular Docking Show Mitocurcumin can Potentially Block Innate Immune Evasion Mechanism of SARS-CoV-2 and Enhance Viral Load Clearance
}

\author{
Debojyoti Pal $^{1}$, Rahul Checker ${ }^{1}$, Vijay K. Kutala ${ }^{2}$ and Santosh K. Sandur ${ }^{1, *}$ \\ ${ }^{1}$ Radiation Biology \& Health Sciences Division, Bio-science Group, Bhabha Atomic Research \\ Centre, Trombay, Mumbai-400085, India \\ ${ }^{2}$ Department of Clinical Pharmacology \& Therapeutics, Nizam's Institute of Medical Sciences, \\ Hyderabad, Telangana, India
}

\section{*Corresponding author}

Dr. Santosh K. Sandur

Radiation Biology \& Health Sciences Division

Bhabha Atomic Research Centre

Trombay, Mumbai, India.

Email: sskumar@barc.gov.in

Tel: 91-22-25595356, Fax: 91-22-25505151 


\section{ABSTRACT:}

The coronavirus disease 19 (COVID-19) is an infectious disease caused by severe acute respiratory syndrome coronavirus 2 (SARS-CoV-2). COVID-19 is affecting human life in an unprecedented manner and has become a public health emergency worldwide. COVID-19 pandemic has prompted numerous researchers worldwide to look for a potential cure on an emergency basis and identification of novel inhibitors of viral infection/replication is the utmost priority to curtail COVID-19 progression. Computational studies have shown curcumin to be a candidate inhibitor of certain SARS-CoV-2 proteins, however, poor bio-availability of curcumin limits its application as a possible therapeutic intervention. In the present work, we have employed a molecular docking approach to study the ability of mitocurcumin (MC), a triphenyl phosphonium conjugated curcumin derivative, to inhibit SARSCoV-2 infection. Computational analysis revealed that MC can bind strongly to SARS-CoV-2 ADP Ribose Phosphatase (NSP3) with high binding energy of $-10.3 \mathrm{kcal} / \mathrm{mol}$ and to SARS-CoV-2 methyltransferase (NSP10-NSP16 complex) with a high binding energy of $-10.4 \mathrm{kcal} / \mathrm{mol}$. We found that MC interacts with critical residues of viral NSP3 macro-domain, known to suppress host immune response, through hydrophobic interactions and occupies its active site. Furthermore, MC interacts with the critical residues of NSP10-NSP16 complex, known to prevent innate immune detection of viral mRNA, through hydrophobic and hydrogen bond interaction and occupies the methyl group donor site. MC is also found to bind to main protease of SARS-CoV-2 and may potentially act as an inhibitor of the viral protease. In conclusion, MC can potentially inhibit the activity of multiple SARSCoV-2 proteins and may accentuate the innate immune system mediated clearance of viral load resulting in improved clinic outcome in COVID-19 patients.

Keywords: SARS-CoV-2, COVID-19, coronavirus, curcumin, Mitocurcumin, docking studies 


\section{INTRODUCTION:}

Severe acute respiratory syndrome coronavirus 2 (SARS-CoV-2) is a novel strain of Severe Acute Respiratory Syndrome-related coronavirus (SARS-CoV) belonging to family Coronaviridae. Coronavirus are enveloped, positive-sense, single-stranded RNA viruses and are characterized by spiky projections that resemble crowns. Four human coronaviruses are globally endemic and known to cause mild to moderate symptoms of cold, accounting for $10-30 \%$ of upper respiratory tract infections globally $(1,2)$. On the other hand, novel coronavirus strains, possibly of zoonotic origin, pose the major threats to human life due to lack of pre-existing immunity allowing the virus to escape innate immune responses.

SARS-CoV-2, which belongs to betacoronavirus strain and is likely of zoonotic origin (3), has wreaked havoc by claiming more than 390,000 lives and infecting over 6.7 million individuals. The basic reproduction number (R0) of the virus has been estimated to be between 1.4 and $3.9(4,5)$, which has led to the rapid spread of the disease. On $30^{\text {th }}$ January 2020, SARS-CoV-2 was designated a Public Health Emergency of International Concern by the WHO and on $11^{\text {th }}$ March 2020 it was declared by WHO as a pandemic. Under these unprecedented conditions, the scientific community is focused on discovering a cure for this disease. A major roadblock in finding a cure for SARS-CoV-2 is its high infectivity and the requirement of stringent handling protocols, which makes direct testing of candidate molecules a difficult proposition. Fortunately, a number of SARS-CoV-2 protein structures have been resolved and are availablein public domain. This makes in silico molecular docking a viable alternative to test the effectiveness of candidate molecules in binding and altering the functionality of critical SARS-CoV-2 proteins before translating to in vitro or in vivo models.

Currently there are no clinically approved antiviral drugs with proven efficiency for SARSCoV-2, except Remdesivir for which Food and Drug Administration (FDA) issued an Emergency Use Authorization (EUA) to be used for the treatment ofhospitalized COVID-19 patients. Hence, 
identification of small molecule inhibitors that can bind to and inactivate viral proteins is an exciting strategy for tackling SARS-CoV-2 infection (6-8). In this direction, curcumin, an active ingredient of the celebrated Ayurvedic herb turmeric, which is well documented to possess antiviral properties against a host of viral pathogens can be a viable pharmacological strategy to combat the virus (9-11). It has been found to work through a repertoire of anti-viral mechanisms including inhibition of binding of enveloped viruses (9), interference of viral replication machinery or suppression of cellular signaling pathways essential for viral replication $(12,13)$.

There are a few unpublished reports who have indeed demonstrated in silico binding of curcumin to SARS-CoV-2 spike protein and its cognate human target ACE2. Unfortunately, despite having afavorable predicted binding affinity, curcumin suffers due to its poor bio-availability $(14,15)$ and multiple strategies are being developed to enhance the bio-availability of curcumin (15). Mitocurcumin (MC), a derivative of curcumin, was synthesized by conjugating curcumin with triphenyl phosphonium (16) resulting in enhanced stability and uptake inside cells as compared to curcumin. MC has been demonstrated to be 25-50 times more effective than curcumin in killing A549 lung cancer cells (17). It has been shown to have anti-bacterial properties at micromolar concentrations (18) which is much superior and physiologically achievable as compared to curcumin. Moreover, the triphenyl phosphonium ring provides a different structural feature and might be helpful in conferring stability by favorable lodging at hydrophobic pockets.

Molecular docking algorithm has made tremendous inroads in the last decade with respect to speed and accuracy, and is considered an appropriate strategy to study the potential candidates before moving to experimental validation. Our molecular docking data indicates that MC can be a promising candidate for the clinical management of COVID-19 patients if the docking results can be translated to clinic. In the present study, we have employed molecular docking to study the binding affinity of MC and its parent molecule curcumin against a wide range of SARS-CoV-2 proteins. Hence, this study provides insight into the putative application of $\mathrm{MC}$ as a small molecule inhibitor for SARS-CoV-2 
proteins as well as its ability to serve as a potential drug to inhibit SARS-CoV-2 infection and replication in the host.

\section{Material and Methods:}

Target selection and structure extraction: SARS-CoV-2 proteins involved in replication, host cell binding, protein processing and evading immune responses were considered to be potential primary targets for small molecule inhibitors. The following structures were obtained from protein data bank: 7BQY (Main Protease), 6M3M (Nucleocapsid), 7BV2 (RNA dependent RNA polymerase; Rdrp), 6WJT (NSP10-NSP16 complex; methyltransferase), 6W02 (NSP3; ADP Ribose Phosphatase), 6W01 (NSP15; endoribonuclease) and 6M0J (Spike protein and human ACE2).

Ligand Preparation: Curcumin and MC were drawn in ACD ChemSketch. DG-AMMOS was used to generate respective 3D conformers (19). Ligands were imported in AutoDock Tools and prepared for docking by assigning the correct AutoDock 4 atom types, subsequently adding Gasteiger charges, merging non-polar hydrogens, detecting aromatic carbons, and setting up the 'torsion tree'.

Molecular Docking Analysis and visualization: Molecular docking was performed using Autodock Vina(20). PDB structures were imported in AutoDock Tools and prepared for rigid docking. $\mathrm{H}_{2} \mathrm{O}$, ions and pre-existing ligands were removed from the structure to free up the active site wherever necessary. Charges were computed, non-polar hydrogens were merged and then the file were exported in pdbqt format. Grid box module was used to set the site of docking. For spike protein, ACE2 and RNA dependant RNA polymerase, multiple sites were chosen for docking. Site of docking were chosen from pre-existing literature or from pre-existing ligands bound to the structure (Table 1). Grid sizes were kept at maximum of 27000 cubic Angstrom. Docking was performed at default exhaustiveness which is calculated depending on the complexity of the ligand and structure. 
Table 1: Description of sites of docking and grid sizes

\begin{tabular}{|c|c|c|c|}
\hline $\begin{array}{l}\text { Serial } \\
\text { number }\end{array}$ & Site name & Description & $\begin{array}{l}\text { Grid Size } \\
\text { (Angstrom) }\end{array}$ \\
\hline 1 & Main Protease & Active site with bound inhibitor N3 & $30 * 30 * 30$ \\
\hline 2 & Spike Protein Site 1 & $\begin{array}{l}\text { Site of salt bridge between K417 (SP) and D30 } \\
\text { (ACE2) }\end{array}$ & $30 * 30 * 30$ \\
\hline 3 & Spike Protein Site 2 & $\begin{array}{l}\text { Site of hydrogen bond between N487 (SP) and } \\
\text { Q24 (ACE2) }\end{array}$ & $30 * 30 * 30$ \\
\hline 4 & Spike Protein Site 3 & $\begin{array}{l}\text { Site of hydrogen bond between Y449 (SP) and } \\
\text { Q42 (ACE2) }\end{array}$ & $30 * 30 * 30$ \\
\hline 5 & $\begin{array}{c}\text { ADP Ribose } \\
\text { Phosphatase (NSP3) }\end{array}$ & Active site with bound ADP ribose & $30 * 30 * 30$ \\
\hline 6 & Nucleocapsid & $\begin{array}{l}\text { Pocket formed by 48-NNTA- } \\
\text { 51,Y110,Y112,T55,A56,R89 }\end{array}$ & $22 * 22 * 22$ \\
\hline 7 & $\begin{array}{l}\text { Endoribonuclease } \\
\qquad(\mathrm{NSP} 15)\end{array}$ & $\begin{array}{l}\text { Pocket formed by H235, H250, K290, S294, } \\
\text { T341, Y343 }\end{array}$ & $30 * 30 * 30$ \\
\hline 8 & Rdrp Site 1 & RNA binding site & $30 * 30 * 30$ \\
\hline 9 & Rdrp Site 2 & NSP12-NSP7 connection & $30 * 30 * 30$ \\
\hline 10 & Rdrp Site 3 & NSP12-NSP8 connection & $30 * 30 * 30$ \\
\hline 11 & $\begin{array}{l}\text { Methyltransferase } \\
\text { (NSP16-NSP10) }\end{array}$ & Active site with bound S-Adenosyl-Methionine & $22 * 30 * 36$ \\
\hline 12 & ACE2 Site 1 & $\begin{array}{l}\text { Site of salt bridge between K417 (SP) and D30 } \\
\text { (ACE2) }\end{array}$ & $30 * 30 * 30$ \\
\hline 13 & ACE2 Site 2 & $\begin{array}{l}\text { Site of hydrogen bond between N487 (SP) and } \\
\text { Q24 (ACE2) }\end{array}$ & $30 * 30 * 30$ \\
\hline 14 & ACE2 Site 3 & $\begin{array}{l}\text { Site of hydrogen bond between Y449 (SP) and } \\
\qquad \text { Q42 (ACE2) }\end{array}$ & $30 * 30 * 30$ \\
\hline
\end{tabular}

After docking, the docked poses were visualized and those having very high binding affinity but poor orientation with respect to active site were discarded. Highest affinity pose with proper binding site 
orientation were reported. Poses with very high binding affinity were visualized with LigPlot+ to elucidate the type of interaction at the binding site (21).

Sequence analysis and alignment: Protein sequences were extracted from Uniprot/PBD and aligned using pBlast module of NCBI Blast. Aligned sequences were visualized with LAST webservice.

\section{RESULTS AND DISCUSSION:}

\section{Mitocurcumin binds to active site of NSP3 with high affinity and may prevent NSP3 mediated} suppression of anti-viral cytokines: NSP3 is the largest protein encoded by the coronavirus genome with its functions ranging from replication, transcription andnucleocapsid binding to immune evasion (22). The macrodomain1, also known as NSP3b is known to play essential role in countering host innate immune response.

MC was predicted to bind to ADP ribose binding site of NSP3 with a very high binding affinity of $-10.3 \mathrm{kcal} / \mathrm{mol}$ (Table 2), which corresponds to a predicted $\mathrm{Ki}$ of $28 \mathrm{nM}$. MC sits in the ADP-ribose binding pocket (Fig 2A and 2B) and interacts primarily through hydrophobic interactions (Fig 2C). The triphenyl groups at the two ends are stabilized by interaction with Gly47, Val 49, Gly 58, Pro 125, Gly 130, Val 155 at one end and by interaction with Asn 99, Val 77, Val 41, Asn 40, Tyr 42, Pro 74 at the other end. The core part is stabilized by Lys44, Phe 132, Ile 131, Gly 46, Ala 50, Ala 38. The presence of triphenyl groups seems to confer the added stability compared to curcumin $(-8.4 \mathrm{kcal} / \mathrm{mol})$.

It has been demonstrated that ADRP-deficient macrodomains displayed an increased sensitivity to the antiviral effect of alpha interferon (23). NSP3's de-ADP-ribosylation activity is essential for evading host immune responses and mutants deficient in ADRP activity showed early and enhanced interferon (IFN), interferon-stimulated gene (ISG), and inflammatory cytokine release. It has also been proposed that de-poly-ADP-ribosylation or de-mono-ADP-ribosylation activities of NSP3 might also be playing an important role in immune evasion. The loss of function mutations in the 
ADRP domain have resulted in reduced viral load in mice (24). Hence, blocking the active site is expected to have the same effect as the function deficient mutations. We had also carried out pairwise sequence alignment of the macrodomain of NSP3 from SARS-CoV-2 and SARS-CoV (Fig 1). Sequences showed 124/168 (74\%) identity and 144/168 (85\%) positive matches. The catalytically essential residues were conserved between SARS-CoV-2 and SARS-COV.

Binding of MC to NSP3 can potentially block its enzymatic activity and thereby prevent SARSCoV-2 from downregulating the anti-viral interferon response. This can lead to enhanced viral load clearance by innate immune system and reduce the extent of viral pathogenesis in individuals without antigen memory i.e. first-time affected individuals.

Mitocurcumin was also found to bind to the active site of NSP10-NSP16 complex and may prevent NSP16 mediated camouflaging of viral RNA strand: Viral RNA recognition through RIGI and IFIT family of proteins is the major mechanism through which the anti-viral pathways are induced in response to foreign RNA in cytoplasm $(25,26)$. RIG-I is a major stimulant of anti-viral interferon production. The basis of detection of viral RNA as 'foreign'lies in the 5' cap structural modifications. Specifically, N-7 and 2'-O positions of the 5' guanosine cap are crucial as these remain methylated in eukaryotic RNA. IFIT1 is an interferon stimulated gene which senses lack of 2'-Omethylation and inhibits viral RNA (27). Viruses in turn mimic this modification by similarly modifying their 5' cap ends using 2'-O-methyltransferase activity and this allows it to evade the immune system (28).

MC binds to the S-Adenosylmethionine binding site of NSP16 (Fig 3A and 3B) with a very high affinity of $-10.4 \mathrm{kcal} / \mathrm{mol}$, which translates to a predicted $\mathrm{Ki}$ of $23 \mathrm{nM}$ (Table 2). It interacts through a mix of hydrogen bonds and hydrophobic interactions (Fig 3C). Hydrogen bonds through Cys6913, Tyr6930 interact with the central core part of MC. The triphenyl moieties are stabilized by hydrophobic interactions with Phe 6947, Leu 6898, Lys 6933, Asp 6931, Asp 6897, Lys 6944 on one end and by 
His 6972, Thr 6970, Ser 6998, Ser 6999, Glu 7001, Lys 6935 on the other end. Coronaviruses possess 2'-O-methyltransferase activity in the NSP16 protein (29) which allows the viral RNA to evade innate immune system detection. NSP16 works in concert with NSP10 and uses S-Adenosylmethionine as the methyl group donor to modify their 5' cap by 2'-O-methylation. The binding of $\mathrm{MC}$ to the $\mathrm{S}$ Adenosylmethionine binding site would deprive NSP16 of the essential methyl-group donor and thus prevent the 2'-O-methyltransferase activity. In absence of 2'-O-methyl group attachment to the 5'cap, viral RNA would get detected by host immune responses leading to enhanced clearance of viral load.

Mitocurcumin binds to viral targets with a higher affinity as compared to curcumin: MC was found to bind to main protease of SARS-CoV-2 with a predicted binding affinity of $-8.4 \mathrm{kcal} / \mathrm{mol}$ and hence may also act as an inhibitor of the same (Fig 4A and 4B). The binding is stabilized by hydrogen bond with Ser 46 and hydrophobic interactions with Thr 25, Met 49, Leu 26, His 41, Cys 145, Asp 187, His 163, Gln 189, Leu 50, Glu 166, Leu 167, Pro 168, Ala 191, Thr 190, Glu 47. Importantly, interaction with catalytically active residues Cys 145 and His 41 means that it can potentially inhibit the activity of Main Protease (Fig 4C).

MC was also found to interact with active site of other SARS-CoV-2 proteins with varying degrees of affinity (Table 2). MC could bind to spike protein salt bridge site $(-5.9 \mathrm{kcal} / \mathrm{mol})$, ACE2 salt bridge site $(-5.8 \mathrm{kcal} / \mathrm{mol})$, nucleocapsid $(-7.2 \mathrm{kcal} / \mathrm{mol})$, NSP15 (-6.8 kcal/mol), Rdrp RNA binding site ($6.7 \mathrm{kcal} / \mathrm{mol})$. It must be noted that in most cases, the affinity of MC was greater than the parent molecule curcumin, probably due the tendency of triphenyl groups to get lodged in hydrophobic pockets and confer extra stability. Based on these observations, MC appears to be a better choice than curcumin as an anti-viral molecule against SARS-CoV-2 infection.

CONCLUSIONS: Our docking studies show that MC can potentially bind and downregulate ADP Ribose phosphatase activity of NSP3, 2'-O-methyltransferase activity of NSP16 as well as the activity of SARS-CoV-2 main protease. The interaction with NSP3 and NSP16 may counteract two crucial 
immune evasions strategies of SARS-CoV-2, rendering it vulnerable to host innate immune response mediated clearance. The nanomolar concentrations, at which MC is predicted to act, would be achievable physiologically making this a realistic candidate molecule against SARS-CoV-2 infection and COVID-19.

\section{Conflict of interest}

All the authors declare that they have no conflicts of interest with the contents of this article. 
Table 2: Predicted binding affinity and Ki values of MC and Curcumin against SARS-CoV-2 proteins

\begin{tabular}{|c|c|c|c|c|c|}
\hline & \multicolumn{2}{|c|}{$\begin{array}{c}\text { Predicted } \\
\text { binding energy } \\
(\text { kcal/mol })\end{array}$} & \multicolumn{2}{|c|}{$\begin{array}{l}\text { Predicted inhibition } \\
\text { constant }(\mu M) \text { at } 298 K(K i \\
=\exp (\operatorname{deltaG} /(R * T))\end{array}$} & \multirow{2}{*}{$\begin{array}{c}\text { Ratio of } \\
\text { predicted } \\
\text { Ki } \\
\text { (Curcumi } \\
\text { n vs MC) }\end{array}$} \\
\hline & $\begin{array}{c}\text { Curcumi } \\
\text { n }\end{array}$ & MC & Curcumin & MC & \\
\hline Main Protease & -6.6 & -8.1 & 14.348 & 1.138 & 12.61 \\
\hline Spike Protein Site & -5.4 & -5.9 & 108.988 & 46.824 & 2.33 \\
\hline $\begin{array}{l}\text { Spike Protein Site } \\
2\end{array}$ & -4.8 & -5.2 & 300.379 & 152.807 & 1.97 \\
\hline $\begin{array}{l}\text { Spike Protein Site } \\
3\end{array}$ & -5.9 & -6.4 & 46.824 & 20.117 & 2.33 \\
\hline $\begin{array}{l}\text { ADP Ribose } \\
\text { Phosphatase } \\
\text { (NSP3) }\end{array}$ & -8.4 & $\begin{array}{l}- \\
10 . \\
3\end{array}$ & 0.685 & 0.028 & 24.47 \\
\hline Nucleocapsid & -5.2 & -7.2 & 152.807 & 5.206 & 29.36 \\
\hline $\begin{array}{l}\text { Endoribonuclease } \\
\text { (NSP15) }\end{array}$ & -5.8 & -6.8 & 55.444 & 10.234 & 5.42 \\
\hline Rdrp Site 1 & -5.7 & -6.7 & 65.65 & 12.118 & 5.42 \\
\hline Rdrp Site 2 & -6.5 & -7.4 & 16.989 & 3.713 & 4.58 \\
\hline Rdrp Site 3 & -6 & -7 & 39.545 & 7.299 & 5.42 \\
\hline $\begin{array}{l}\text { Methyltransferase } \\
\text { (NSP16-NSP10) }\end{array}$ & -7.5 & $\begin{array}{l}- \\
10 . \\
4\end{array}$ & 3.136 & 0.023 & 136.35 \\
\hline ACE2 Site 1 & -5.6 & -5.8 & 77.735 & 55.444 & 1.41 \\
\hline ACE2 Site 2 & -5.2 & -5 & 152.807 & 214.243 & 0.72 \\
\hline ACE2 Site 3 & -5 & -5.8 & 214.243 & 55.444 & 3.87 \\
\hline
\end{tabular}


Alignment score $=655$, expect $=1.1 \mathrm{e}-103$ :

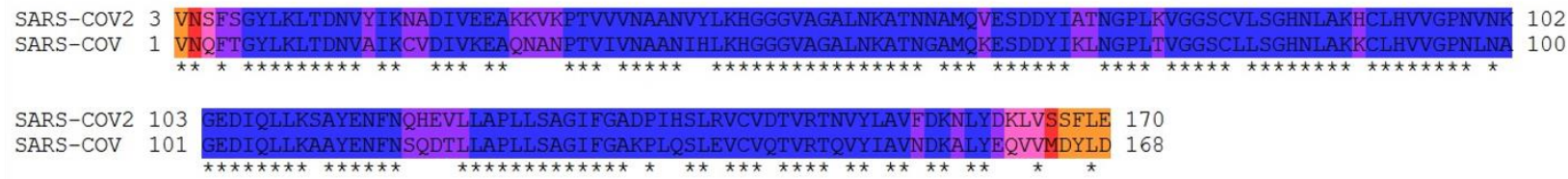

Figure 1: Pairwise sequence alignment of macrodomain of NSP3 from SARS-CoV-2 and SARSCOV. Sequences show 124/168(74\%) identity and 144/168(85\%) positive matches

$2 \mathrm{~A}$

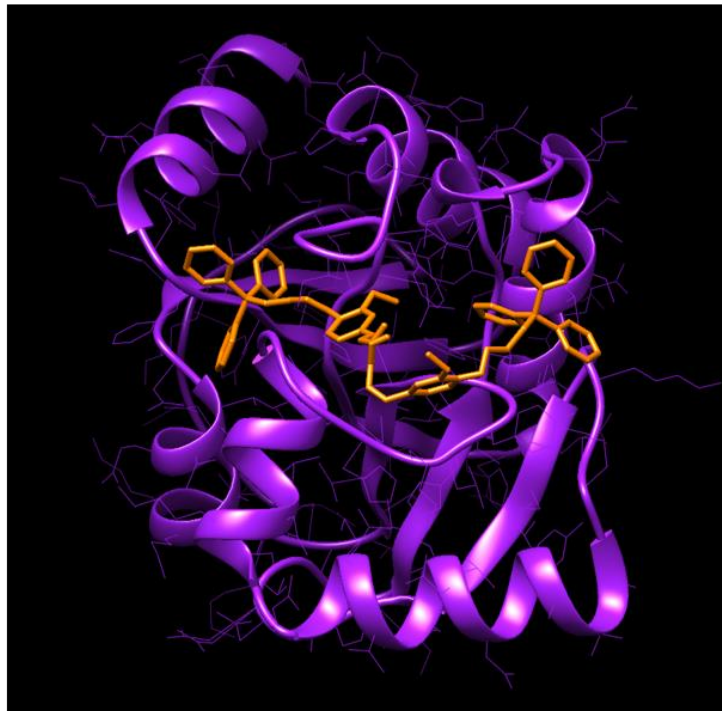

$2 \mathrm{~B}$

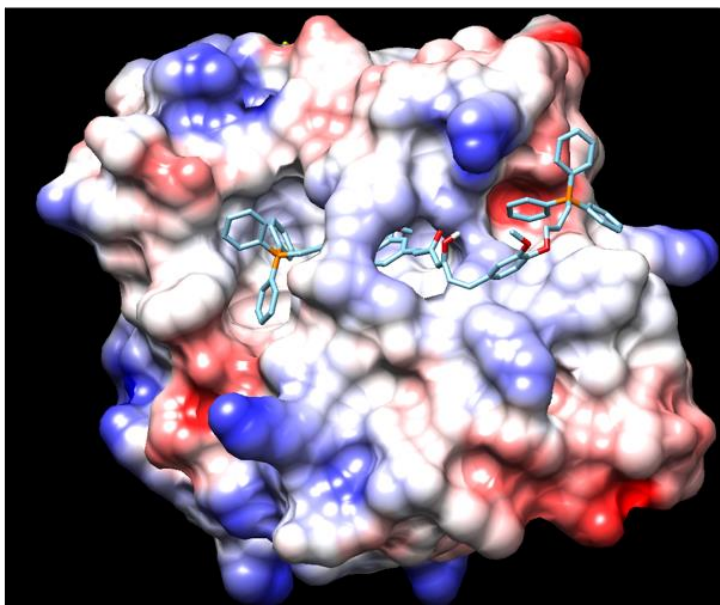

$2 \mathrm{C}$

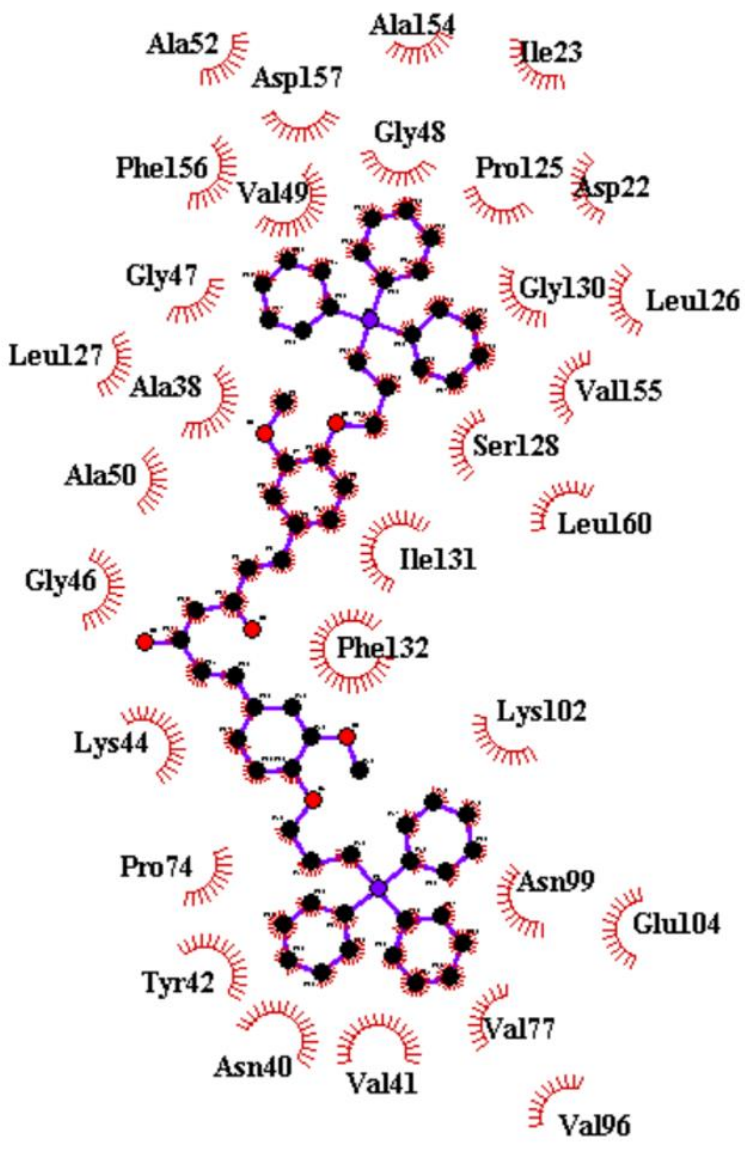

Figure 2: Docked conformation of MC at active site of macrodomain of NSP3 (PDB ID 6W02) represented in (A) ribbon and stick model and (B) surface charge model. (C) 2D representation of various hydrophobic interactions (red semi circles) between MC and NSP3. 

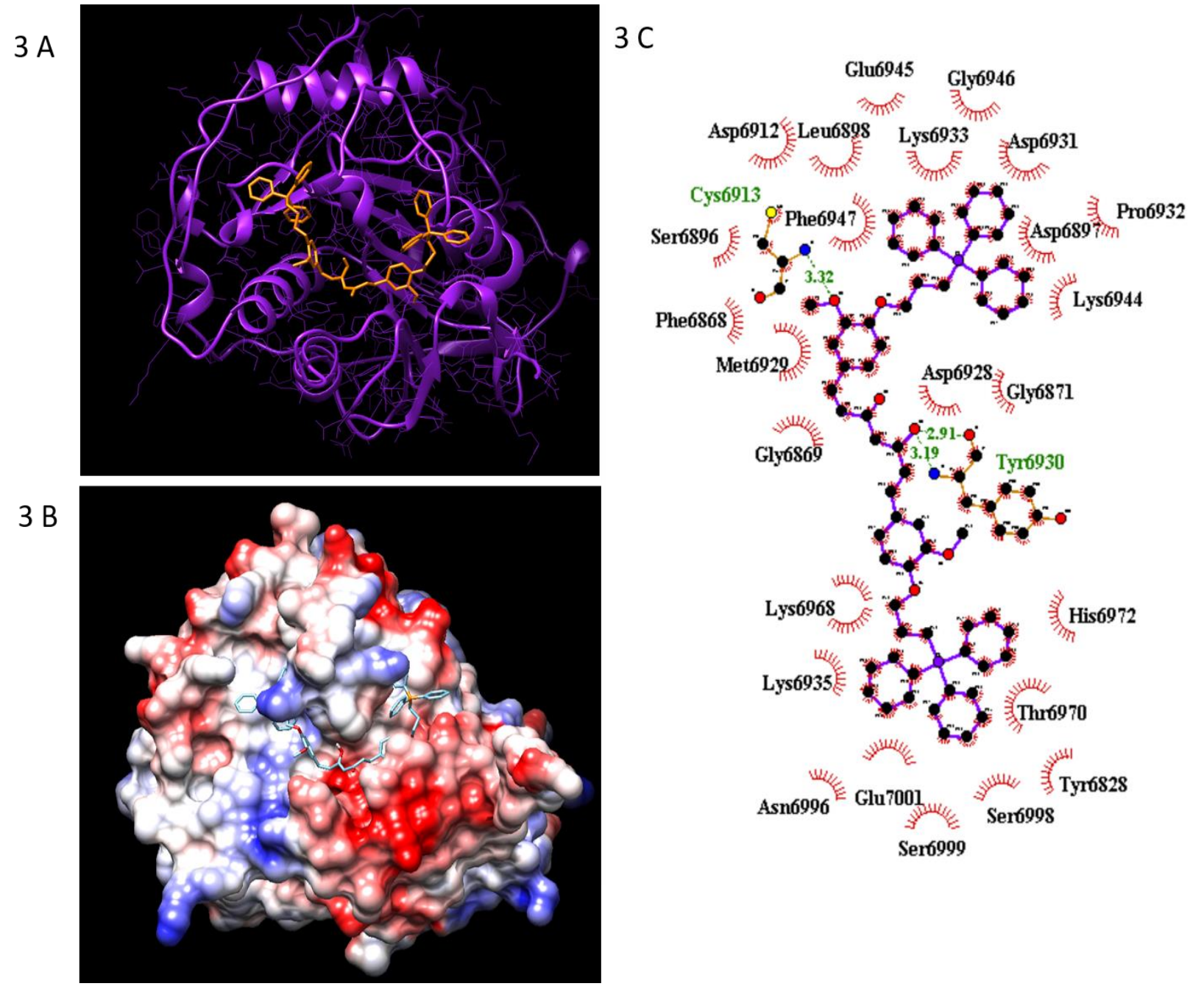

Figure 3: Docked conformation of MC at S-Adenosylmethionine binding site of NSP16 (PDB ID $6 \mathrm{WJT}$ ) represented in (A) ribbon and stick model and (B) surface charge model. (C) 2D representation of various hydrophobic (red semi circles) and hydrogen bond (green lines) interactions between MC and NSP16. Numbers in green indicate H-bond length. 
$4 \mathrm{~A}$

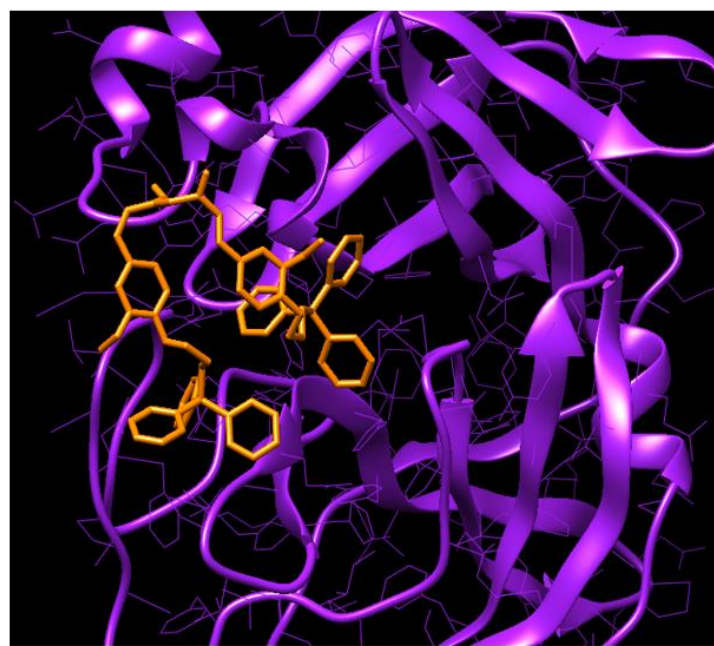

$4 \mathrm{~B}$

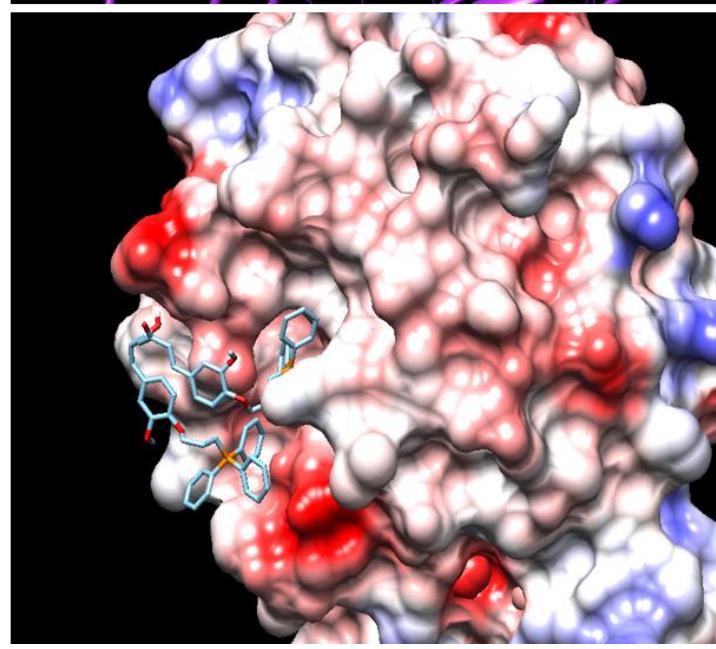

$4 \mathrm{C}$

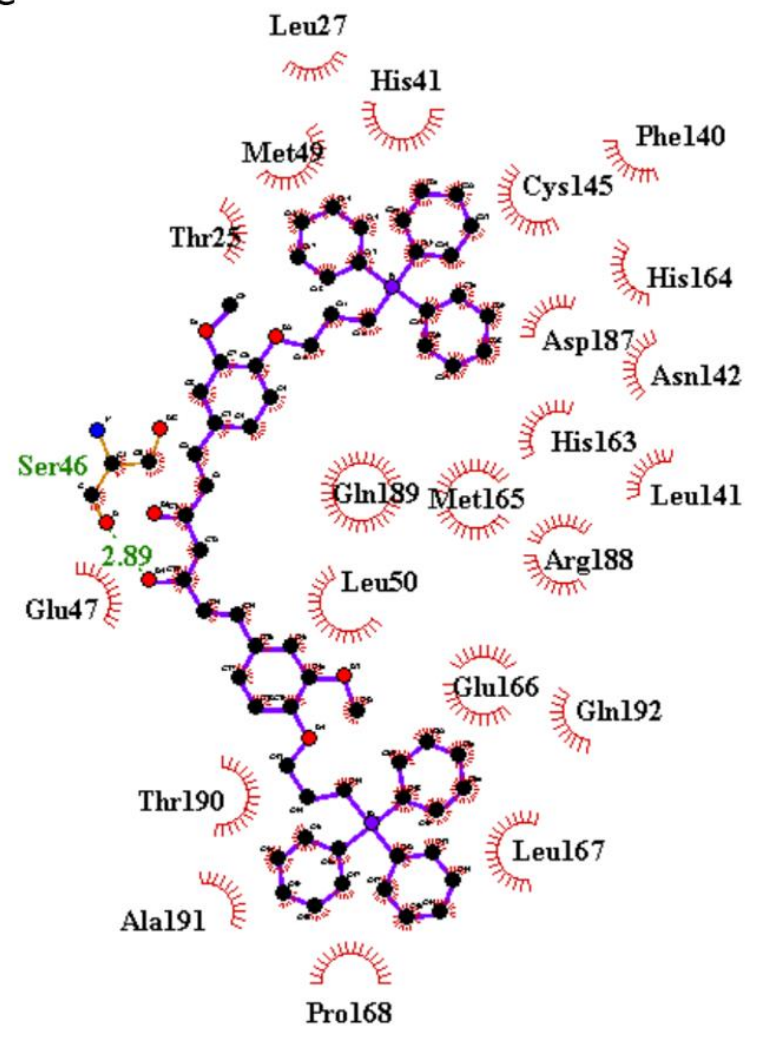

Figure 4: Docked conformation of MC at active site of SARS-CoV-2 main protease (PDB ID 7BQY) represented in (A) ribbon and stick model and (B) surface charge model. (C) 2D representation of various hydrophobic (red semi circles) and hydrogen bond (green lines) interactions between MC and SARS-CoV-2 main protease. Numbers in green indicate H-bond length 


\section{References:}

1. Paules CI, Marston HD, Fauci AS. Coronavirus Infections-More Than Just the Common Cold. JAMA. 2020. Jan 23, 2020;10.1001/jama.2020.0757

2. de Wit E, van Doremalen N, Falzarano D, Munster VJ. SARS and MERS: recent insights into emerging coronaviruses. Nat Rev Microbiol. 2016;14(8):523-34.

3. Andersen KG, Rambaut A, Lipkin WI, Holmes EC, Garry RF. The proximal origin of SARSCoV-2. Nat Med. 2020;26(4):450-2.

4. Li Q, Guan X, Wu P, Wang X, Zhou L, Tong Y, et al. Early Transmission Dynamics in Wuhan, China, of Novel Coronavirus-Infected Pneumonia. N Engl J Med. 2020;382(13):1199-207.

5. Riou J, Althaus CL. Pattern of early human-to-human transmission of Wuhan 2019 novel coronavirus (2019-nCoV), December 2019 to January 2020. Euro Surveill. 2020;25(4).

6. Litterman N, Lipinski C, Ekins S. Small molecules with antiviral activity against the Ebola virus. F1000Res. 2015;4:38.

7. Agostini ML, Pruijssers AJ, Chappell JD, Gribble J, Lu X, Andres EL, et al. Small-Molecule Antiviral beta-d-N (4)-Hydroxycytidine Inhibits a Proofreading-Intact Coronavirus with a High Genetic Barrier to Resistance. J Virol. 2019;93(24).

8. Han J, Perez J, Schafer A, Cheng H, Peet N, Rong L, et al. Influenza Virus: Small Molecule Therapeutics and Mechanisms of Antiviral Resistance. Curr Med Chem. 2018;25(38):5115-27.

9. Mounce BC, Cesaro T, Carrau L, Vallet T, Vignuzzi M. Curcumin inhibits Zika and chikungunya virus infection by inhibiting cell binding. Antiviral Res. 2017;142:148-57.

10. Praditya D, Kirchhoff L, Bruning J, Rachmawati H, Steinmann J, Steinmann E. Anti-infective Properties of the Golden Spice Curcumin. Front Microbiol. 2019;10:912.

11. Zandi K, Ramedani E, Mohammadi K, Tajbakhsh S, Deilami I, Rastian Z, et al. Evaluation of antiviral activities of curcumin derivatives against HSV-1 in Vero cell line. Nat Prod Commun. 2010;5(12):1935-8. 
12. Dony M, Hsu W. Antiviral potential of curcumin. Journal of Functional Foods. 2018;40:8.

13. Moghadamtousi SZ, Kadir HA, Hassandarvish P, Tajik H, Abubakar S, Zandi K. A review on antibacterial, antiviral, and antifungal activity of curcumin. Biomed Res Int. 2014;2014:186864.

14. Nelson KM, Dahlin JL, Bisson J, Graham J, Pauli GF, Walters MA. The Essential Medicinal Chemistry of Curcumin. J Med Chem. 2017;60(5):1620-37.

15. Anand P, Kunnumakkara AB, Newman RA, Aggarwal BB. Bioavailability of curcumin: problems and promises. Mol Pharm. 2007;4(6):807-18.

16. Vasagiri N, Kutala VK. Molecular mechanism of interaction of mitocurcumin-1 with Akt1 and STAT3: an in silico approach. Indian J Biochem Biophys. 2014;51(4):308-13.

17. Jayakumar S, Patwardhan RS, Pal D, Singh B, Sharma D, Kutala VK, et al. Mitochondrial targeted curcumin exhibits anticancer effects through disruption of mitochondrial redox and modulation of TrxR2 activity. Free Radic Biol Med. 2017;113:530-8.

18. Kumari S, Jayakumar S, Gupta GD, Bihani SC, Sharma D, Kutala VK, et al. Antibacterial activity of new structural class of semisynthetic molecule, triphenyl-phosphonium conjugated diarylheptanoid. Free Radic Biol Med. 2019;143:140-5.

19. Lagorce D, Pencheva T, Villoutreix BO, Miteva MA. DG-AMMOS: a new tool to generate 3d conformation of small molecules using distance geometry and automated molecular mechanics optimization for in silico screening. BMC Chem Biol. 2009;9:6.

20. Trott O, Olson AJ. AutoDock Vina: improving the speed and accuracy of docking with a new scoring function, efficient optimization, and multithreading. J Comput Chem. 2010;31(2):455-61.

21. Laskowski RA, Swindells MB. LigPlot+: multiple ligand-protein interaction diagrams for drug disCoVery. J Chem Inf Model. 2011;51(10):2778-86.

22. Lei J, Kusov Y, Hilgenfeld R. Nsp3 of coronaviruses: Structures and functions of a large multidomain protein. Antiviral Res. 2018;149:58-74. 
23. Kuri T, Eriksson KK, Putics A, Zust R, Snijder EJ, Davidson AD, et al. The ADP-ribose-1"monophosphatase domains of severe acute respiratory syndrome coronavirus and human coronavirus 229E mediate resistance to antiviral interferon responses. J Gen Virol. 2011;92(Pt 8):1899-905.

24. Fehr AR, Channappanavar R, Jankevicius G, Fett C, Zhao J, Athmer J, et al. The Conserved Coronavirus Macrodomain Promotes Virulence and Suppresses the Innate Immune Response during Severe Acute Respiratory Syndrome Coronavirus Infection. mBio. 2016;7(6).

25. Leung DW, Amarasinghe GK. When your cap matters: structural insights into self vs non-self recognition of 5' RNA by immunomodulatory host proteins. Curr Opin Struct Biol. 2016;36:133-41.

26. Schuberth-Wagner C, Ludwig J, Bruder AK, Herzner AM, Zillinger T, Goldeck M, et al. A Conserved Histidine in the RNA Sensor RIG-I Controls Immune Tolerance to N1-2'O-Methylated Self RNA. Immunity. 2015;43(1):41-51.

27. Hyde JL, Diamond MS. Innate immune restriction and antagonism of viral RNA lacking 2-O methylation. Virology. 2015;479-480:66-74.

28. Daffis S, Szretter KJ, Schriewer J, Li J, Youn S, Errett J, et al. 2'-O methylation of the viral mRNA cap evades host restriction by IFIT family members. Nature. 2010;468(7322):452-6.

29. Lehmann KC, Hooghiemstra L, Gulyaeva A, Samborskiy DV, Zevenhoven-Dobbe JC, Snijder EJ, et al. Arterivirus nsp12 versus the coronavirus nsp16 2'-O-methyltransferase: comparison of the Cterminal cleavage products of two nidovirus pp1ab polyproteins. J Gen Virol. 2015;96(9):2643-55. 